\title{
ALTERAÇÕES MORFOLÓGICAS E HEMODINÂMICAS TARDIAS NO TERRITÓRIO ESPLÊNICO DE PACIENTES COM ESQUISTOSSOMOSE MANSÔNICA HEPATOESPLÊNICA PÓS-ANASTOMOSE ESPLENORRENAL DISTAL. (Estudo com ultra-som Doppler)
}

\author{
Azzo WIDMAN ${ }^{1}$, Ilka Regina Souza de OLIVEIRA $^{2}$, Manlio Basílio SPERANZINI ${ }^{3}$, \\ Giovanni Guido CERRI $^{2}$, William Abrão SAAD ${ }^{1}$ e Joaquim GAMA-RODRIGUES ${ }^{3}$
}

RESUMO - Racional - A anastomose esplenorrenal distal (operação de Warren) tem sido indicada para tratamento do sangramento digestivo provocado pelas varizes esofágicas pois, idealmente, reduziria a pressão venosa no território cardiotuberositário sem alterar o fluxo mesentérico-portal. Entretanto, as mudanças que esta operação provoca no território esplênico não foram totalmente esclarecidas. Objetivo - Avaliar alterações morfológicas e hemodinâmicas tardias no território esplênico decorrentes da anastomose esplenorrenal distal, em pacientes com hipertensão portal por esquistossomose mansônica hepatoesplênica complicada com hemorragia pelas varizes esofágicas. Método - Estudo, mediante ultra-som-Doppler, da região esplênica de 52 pacientes com hipertensão portal por esquistossomose hepatoesplênica divididos em dois grupos: 40 não-operados e 12 com anastomose esplenorrenal distal. Neles foram comparados: a) parâmetros morfométricos (diâmetro da artéria e veia esplênicas, diâmetros esplênicos: longitudinal, transversal e ântero-posterior); b) parâmetros velocimétricos do fluxo nos vasos esplênicos (velocidade de pico sistólico na artéria esplênica e velocidade média de fluxo na veia esplênica); c) índice biométrico do baço (longitudinal x transversal); d) índice volumétrico do baço (longitudinal x transversal x ântero-posterior x 0,523); e) índices hemodinâmicos de impedância na artéria esplênica (pulsatilidade e resistividade). Resultados - Nos pacientes com anastomose esplenorrenal distal observaram-se: a) redução - índice volumétrico (não-operados $903,83 \pm 452,77 \mathrm{~cm}^{3} /$ anastomose esplenorrenal distal 482,32 $\pm 208,02 \mathrm{~cm}^{3}(46,64 \%)$ ) e biométrico esplênico (não-operados 138,14 $\pm 51,89 \mathrm{~cm}^{2} /$ anastomose esplenorrenal distal $\left.94,83 \pm 39,83 \mathrm{~cm}^{2}(33,35 \%)\right)$; b) ausência de variação - artéria esplênica: diâmetro (não-operados $0,57 \pm 0,16 \mathrm{~cm} /$ anastomose esplenorrenal distal $0,57 \pm 0,23 \mathrm{~cm}$ )), velocidade de pico sistólico na artéria esplênica (não-operados $107 \pm 42,02$ $\mathrm{cm} / \mathrm{seg} /$ anastomose esplenorrenal distal $89,81 \pm 41,20 \mathrm{~cm} / \mathrm{seg}$ )), índice de resistividade (não-operados $0,58 \pm 0,008 /$ anastomose esplenorrenal distal $0,56 \pm 0,06)$ ) e índice de pulsatilidade (não-operados $0,91 \pm 0,19$ /anastomose esplenorrenal distal $0,86 \pm$ $0,15)$; - veia esplênica (não-operados $1,10 \pm 0,30 \mathrm{~cm} /$ anastomose esplenorrenal distal $1,19 \pm 0,29 \mathrm{~cm}$ )); c) aumento - da velocidade média de fluxo na veia esplênica (não-operados $20,54 \pm 8,45 \mathrm{~cm} / \mathrm{seg} /$ anastomose esplenorrenal distal 27,83 $\pm 9,29$ $\mathrm{cm} / \mathrm{seg}$ )). Conclusões - A comparação dos exames ultra-som-Doppler entre os dois grupos (não-operados e anastomose esplenorrenal distal) mostrou que nos pacientes com esquistossomose mansônica hepatoesplênica e operados de anastomose esplenorrenal distal há redução dos índices biométrico e volumétrico do baço; não há modificação dos parâmetros morfológicos e hemodinâmicos nem dos índices velocimétricos da artéria esplênica; há aumento da velocidade média de fluxo na veia esplênica.

DESCRITORES - Baço. Esquistossomose mansoni. Hipertensão portal. Anastomose cirúrgica. Ultra-sonografia Doppler.

\footnotetext{
Trabalho Realizado nos Departamentos de Gastroenterologia e de Radiologia da Faculdade de Medicina da Universidade de São Paulo - FMUSP, São Paulo, SP.

${ }^{1}$ Grupo de Cirurgia do Fígado e Hipertensão Portal da Divisão de Clínica Cirúrgica II do Hospital das Clínicas da Faculdade de Medicina da Universidade de São Paulo - HCFMUSP; ${ }^{2}$ Departamento de Radiologia da FMUSP; ${ }^{3}$ Disciplina de Cirurgia do Aparelho Digestivo do Departamento de Gastroenterologia da FMUSP.
} Endereço para correspondência: Dr. Azzo Widman - Rua Guilherme Moura, 302 - 05449-010 - São Paulo, SP. 


\section{INTRODUÇÃO}

A anastomose esplenorrenal distal (AERD) (operação de Warren) (W) foi proposta para o tratamento da hipertensão portal por cirrose hepática com a finalidade de reduzir a pressão venosa nas varizes esofágicas. Esta operação, que deriva o segmento distal da veia esplênica à veia renal, cria uma via de drenagem portossistêmica setorial, estabelecendo comunicação entre o sistema cardiotuberositário e a veia cava, sem interferir no fluxo mesentérico-portal. Deste modo, as alterações circulatórias produzidas naquele território vascular teriam a finalidade de evitar o sangramento digestivo alto que é fator complicador na evolução clínica da doença ${ }^{(21)}$. Esta técnica terapêutica também foi aplicada em pacientes com hipertensão portal por esquistossomose mansônica hepatoesplênica (EHE) e que tiveram sangramento pelas varizes esofágicas ${ }^{(17)}$.

Entretanto, nesta doença há esplenomegalia mais acentuada do que na cirrose hepática ${ }^{(4)}$, apesar de ambas terem em comum a hipertensão portal e suas complicações ${ }^{(3)}$.

$\mathrm{Na}$ gênese desta visceromegalia concorrem dois fatores cuja influência individual não está bem esclarecida: o hemodinâmico (aporte arterial e congestão venosa) e a hiperplasia da polpa esplênica.

$\mathrm{Na}$ AERD, o aporte arterial é mantido e a facilitação da drenagem do sangue venoso do baço reduz a congestão venosa esplênica. Deste modo, as alterações residuais observáveis nesta víscera após a realização deste tratamento cirúrgico ${ }^{(8,13,20)}$, seriam dependentes da polpa esplênica e têm importância clínica. O escasso comprometimento da função hepática na EHE e a sobrevida prolongada dos pacientes, torna a hipertensão portal desta doença um modelo adequado para avaliar as alterações tardias (morfológicas e hemodinâmicas) que a AERD provoca no setor esplênico.

O ultra-som Doppler (US-Doppler), método não-invasivo de diagnóstico por imagem, é utilizado habitualmente para verificar se a AERD está pérvia ${ }^{(1,14)}$. Entretanto, ele também permite a medida de vários parâmetros: volume dos órgãos sólidos intra-abdominais, diâmetro dos vasos, direção do fluxo sangüíneo e velocidade no interior dos mesmos. Além destas medidas, a análise dos índices que resultam da relação entre os valores obtidos, permite que sejam avaliadas as condições morfológicas e hemodinâmicas do setor estudado. Os índices morfológicos foram validados através de várias associações de diâmetros ("cortes") dos órgãos sólidos, medidos pelo US-Doppler que, ao serem comparadas com o tamanho real do órgão, permitiram a escolha dos que melhor exprimissem esta relação ${ }^{(7,}$ $9,10,11,12,15,18,19)$

O objetivo deste trabalho foi avaliar as alterações tardias que a ERD provoca no território esplênico. Para esta finalidade foram quantificados os aspectos morfológicos e hemodinâmicos desta região em pacientes com EHE com o auxílio da US-Doppler.

\section{CASUÍSTICA E MÉTODO}

Estudaram-se os resultados do exame US-Doppler de 52 pacientes consecutivos provenientes do Ambulatório do Grupo de Cirurgia do Fígado e Hipertensão Portal da Divisão de Clínica Cirúrgica II do Hospital das Clínicas da Faculdade de Medicina da Universidade de São Paulo, SP. Todos apresentavam EHE e história de sangramento digestivo alto por varizes esofágicas.

Os pacientes estavam distribuídos em dois grupos: 40 sem operação (NO) e 12 operados de AERD (W).

Os pacientes NO estavam em fase de exames pré-operatórios e os operados (período superior a 6 meses) estavam sob acompanhamento no ambulatório e realizando exames rotineiros de controle periódico.

Critério de inclusão dos pacientes operados: AERD pérvia.

\section{Método}

Através do US-Doppler foram medidos os seguintes parâmetros: três diâmetros do baço - longitudinal (LGT), transversal (TRV) e ântero-posterior (AP) (7) - ; o diâmetro da artéria e da veia esplênica; a velocidade de pico sistólico (VPS) na artéria esplênica e a velocidade média de fluxo $\left(\mathrm{T}_{\max }\right)$ na veia esplênica. A partir destes resultados, calcularam-se vários índices: o volumétrico (LGT x TRV x AP x 0,523) ${ }^{(7)}$ e o biométrico do baço (LGT x TRV) ${ }^{(10)}$; o de pulsatilidade (IP) e o de resistividade (IR) na artéria esplênica.

Os resultados dos parâmetros e índices foram anotados na forma de média e desvio padrão e comparados entre os dois grupos.

\section{Exame ultra-sonográfico}

Todos os pacientes foram examinados em condições de jejum de 12 horas e ingestão, prévia ao exame, de $10 \mathrm{mg}$ de simeticona $\left(\right.$ Luftal $\left.^{\circledR}\right)$ para reduzir a interferência dos gases intestinais.

Os exames foram realizados em equipamento de ultra-sonografia de tempo real em modo-B com transdutor convexo de 3,5 $\mathrm{MHz}$ (GE - Logic 500 MD, EUA) e Doppler pulsado na freqüência de 3,5 e 2,5 $\mathrm{MHz}$.

Os parâmetros morfométricos do baço, da artéria e veia esplênica foram medidos no modo-B. Em seguida, o feixe Doppler foi direcionado para o vaso estudado com correção do ângulo ao feixe Doppler e posicionada a amostra de volume (cerca de $2 / 3$ do calibre) na luz do mesmo. A $\mathrm{T}_{\max }$ da veia esplênica, assim como a VPS e os índices IP e IR da artéria esplênica foram calculados automaticamente pelo "software" específico do equipamento (operador-independente) através do traçado espectral. 


\section{Tratamento estatístico}

A comparação dos resultados de parâmetros e índices dos dois grupos de pacientes foi feita mediante análise de variância $(P<0.05$ : significativo).

\section{RESULTADOS}

Todos os pacientes com AERD (W) apresentavam a anastomose pérvia e os resultados do exame US-Doppler, quando comparados aos dos pacientes NO mostraram que:

a) houve redução dos três diâmetros (LGT, TRV e AP) do baço, do índice volumétrico e biométrico esplênico;

b) não houve alteração do diâmetro, da VPS, dos índices de resistividade (IR) e de pulsatilidade (IP) na artéria esplênica; do diâmetro na veia esplênica;

c) houve aumento da $T_{\max }$ na veia esplênica.

Todos os parâmetros e índices avaliados no território esplênico dos doentes estão representados nas Tabelas 1 e 2.

\section{COMENTÁRIOS}

\section{Volume esplênico}

Comparando os resultados da medida US-Doppler do baço dos doentes com EHE aos dos pacientes normais, verificou-se que nos operados houve redução estatisticamente significativa em todos os diâmetros medidos e, conseqüentemente, dos índices volumétrico e biométrico (Tabela 1).

A grande variedade de tamanho do baço, observada nos doentes com EHE NO e representada pelo desvio padrão no cálculo da média (Tabela 1), dificulta a comparação dos dois grupos de doentes por dois fatos: pacientes não-pareados e reduzido número dos pacientes operados. Entretanto, a análise estatística dos resultados, mostrando significância, permite inferir a possibilidade de comparar os dois grupos.

A avaliação clínica da esplenomegalia pela palpação e percussão, após a realização de AERD em pacientes com EHE, mostrou resultados conflitantes: ausência de variação ${ }^{(20)}$ e redução volumétrica não especificada $^{(8,13)}$. Entretanto, quando a medida foi feita na fase venosa da angiografia esplênica, foi notada redução de aproximadamente $40 \%$ do volume, o que é comparável aos resultados que se obteve pelo exame US-Doppler dos doentes desta série.

As eventuais disparidades dos resultados da medida do baço por vários métodos podem ser devidas à forma irregular do órgão. $\mathrm{O}$ exame clínico (percussão e palpação) é operador dependente e pouco confiável, pois o órgão se situa no hipocôndrio esquerdo e é protegido pelo gradeado costal, tornando-se perceptível somente quando seu diâmetro longitudinal aumenta ${ }^{(10,15,16)}$. Os métodos de imagem invasivos: radiografia ${ }^{(16)}$, angiografia ${ }^{(2)}$, tomografia computadorizada e radioisotopia ${ }^{(22)}$, ainda que apresentem bom índice de correlação com o tamanho físico do órgão ${ }^{(6)}$, expõem os pacientes à radiação e ao uso dos contrastes. A US-Doppler, apesar de se valer de índices ${ }^{(7,10)}$, pode

TABELA 1 - Parâmetros US-Doppler do baço e índices morfométricos

\begin{tabular}{lcccccc}
\hline & n & Baço LGT & Baço TRV & Baço AP & Índice & $\begin{array}{c}\text { Índice } \\
\text { bolumétrico }\end{array}$ \\
\hline NO & 40 & $17,99 \pm 3,48$ & $7,49 \pm 1,57$ & $12,12 \pm 2,28$ & $903,83 \pm 452,77$ & $138,14 \pm 51,89$ \\
W & 12 & $14,00 \pm 3,09$ & $6,06 \pm 2,06$ & $9,76 \pm 1,80$ & $482,32 \pm 208,02$ & $94,83 \pm 39,83$ \\
Total & 52 & $22,18 \%$ & $19,1 \%$ & $19,47 \%$ & $46,64 \%$ & $33,35 \%$ \\
& & $\left(P=0,000^{*}\right)$ & $P=0,013^{*}$ & $P=0,002^{*}$ & $P=0,003 *$ & $P=0,011^{*}$ \\
\hline
\end{tabular}

$\mathrm{NO}=$ não-operados; $\mathrm{W}$ = anastomose esplenorrenal (AERD); LGV = diâmetro longitudinal;

TRV = diâmetro transversal; $\mathrm{AP}=$ diâmetro ântero-posterior; $*=P$ significativo $(<0,05)$

TABELA 2 - Parâmetros hemodinâmicos US-Doppler e índices da circulação esplênica

\begin{tabular}{|c|c|c|c|c|c|c|c|}
\hline & $\mathrm{n}$ & $\begin{array}{c}\text { Diâmetro } \\
\mathbf{A E}\end{array}$ & $\begin{array}{c}\text { VPS } \\
\mathbf{A E}\end{array}$ & $\begin{array}{c}\text { Diâmetro } \\
\text { VEh }\end{array}$ & $\begin{array}{l}T_{\max } \\
\text { VEh }\end{array}$ & $\begin{array}{l}\mathbf{I P} \\
\mathbf{A E}\end{array}$ & $\begin{array}{l}\text { IR } \\
\mathbf{A E}\end{array}$ \\
\hline $\mathrm{NO}$ & 40 & $0,57 \pm 0,16$ & $107,36 \pm 42,02$ & $1,10 \pm 0,30$ & $20,54 \pm 8,45$ & $0,91 \pm 0,19$ & $0,58 \pm 0,08$ \\
\hline W & 12 & $0,57 \pm 0,23$ & $89,87 \pm 41,20$ & $1,19 \pm 0,29$ & $27,83 \pm 9,29$ & $0,86 \pm 0,15$ & $0,56 \pm 0,06$ \\
\hline Total & 52 & $P=1,000$ & $P=0,209$ & $P=0,363$ & $P=0,013^{*}$ & $P=0,408$ & $P=0,428$ \\
\hline
\end{tabular}

$\mathrm{NO}=$ não operados; $\mathrm{W}=$ anastomose esplenorrenal (AERD); $\mathrm{AE}=$ artéria esplênica;

VPS = velocidade de pico sistólico na artéria esplênica; Veh = veia esplênica no hilo; IP = índice de pulsatilidade;

IR = índice de resistividade; $\mathrm{T}_{\max }=$ velocidade média de fluxo na veia esplênica; $*=P$ significativo $(<0,05)$ 
ser utilizada para avaliar e comparar volumes esplênicos e tem a vantagem de não ser invasiva.

Diferentemente do constatado no presente estudo, não foi observada diferença estatisticamente significativa do único diâmetro (LGT) medido pelo exame com US-Doppler ${ }^{(5)}$ em pacientes cirróticos operados de AERD e NO. Este diâmetro, bem como os demais e também os índices adotados nesta casuística, apresentaram diminuição apreciável nos doentes desta série (Tabela 1).

A diminuição mais acentuada do índice volumétrico $(46,64 \%)$ do que do biométrico $(33,35 \%)$ pode ser atribuída à diferença do modo de calcular seus valores. Entretanto, ambos os índices indicam que houve queda acentuada do tamanho do órgão após a realização da operação.

Interpreta-se este fato como sendo o resultado do efeito hemodinâmico da AERD que, facilitando o escoamento do sangue do baço, eliminou o fator congestão venosa e reduziu o tamanho do órgão.

A mensuração do tamanho do baço, após ter sido eliminada a congestão venosa no território esplênico pela AERD, tornou possível a avaliação da repercussão do componente hemodinâmico venoso sobre a esplenomegalia de longa duração na EHE.

Entretanto a interpretação das discrepâncias fica dificultada pelo número reduzido de estudos realizados e pela falta de homogeneização dos parâmetros e índices estudados.

\section{Velocidade de pico sistólico na artéria esplênica (VPS)}

Este parâmetro, nunca antes avaliado no setor esplênico de pacientes com EHE ou cirrose hepática, representa o regime circulatório arterial do baço.

Observou-se VPS menor nos pacientes operados, mas que não foi estatisticamente significativa (Tabela 2 ). O pequeno número de pacientes examinados $(\mathrm{n}=12)$ na presente casuística e a falta de significância estatística da diferença numérica entre ambos os grupos, torna difícil a análise deste resultado. Entretanto, se esta tendência à diminuição for comprovada, indicaria que o tratamento cirúrgico, ainda que facilitando o escoamento venoso, paradoxalmente reduziria o ritmo circulatório no órgão.

O aumento do número de estudos deste parâmetro US-Doppler em pacientes com EHE e operados de AERD, assim como sua avaliação em outras doenças que tenham resultado em hipertensão portal, talvez torne possível estabelecer seu significado fisiopatológico.

\section{Índices de impedância na artéria esplênica}

O IR e IP exprimem as condições circulatórias do leito vascular intraparenquimatoso do baço. Estando relacionados com a complacência das paredes vasculares arteriais e com a resistência orgânica ao fluxo, indicam a dificuldade à passagem do sangue pelo leito capilar.
Não se encontrou diferença estatisticamente significativa nos valores destes índices quando se compararam os dois grupos de doentes (Tabela 2). $\mathrm{Na}$ EHE, a redução dos parâmetros e índices morfométricos do baço nos pacientes operados de AERD (Tabela 1), pressupõe o adensamento tecidual no órgão, pela remoção da congestão venosa esplênica, com conseqüente aumento da resistência capilar e dos índices de impedância. Entretanto, a falta de mudança nos valores dos índices de impedância arterial, sugere que na EHE a retirada da dificuldade de drenagem venosa não intervém nas condições hemodinâmicas da circulação arteriovenosa esplênica.

$\mathrm{Na}$ cirrose hepática foram observados valores significativamente reduzidos destes índices, nos pacientes em que havia alguma forma de derivação portossistêmica que permitisse a descongestão esplênica (anastomose portocava, esplenorrenal distal e derivação portossistêmica transjugular (TIPPS) $)^{(5)}$. Nestes doentes, não foi constatada a redução das dimensões do órgão, devendo-se, no entanto, levar em conta o fato de que naquela casuística foi mensurado um único parâmetro morfométrico do baço (LGT). Esta situação leva a concluir que a facilidade de drenagem do fluxo venoso esplênico na cirrose hepática propicia a redução da resistência capilar à circulação transesplênica.

A discrepância de resultados entre os pacientes com cirrose hepática e EHE, em que pese haver somente um estudo ${ }^{(5)}$, sugere que haja diferenças de resposta biológica esplênica a estas doenças.

\section{Velocidade média de fluxo na veia esplênica $\left(\mathbf{T}_{\max }\right)$}

O sangue da veia esplênica tem várias origens, sendo o baço a mais importante e em condições normais é drenado para a veia porta. Entretanto, a dificuldade de drenagem transhepática do sangue do território digestivo, na hipertensão portal, promove a congestão esplênica e as conexões anastomóticas naturais entre os sistemas cardiotuberositário com os vasos gástricos breves, gastroomental da esquerda e mesentérico (veia mesentérica inferior), adquirem importância hemodinâmica e clínica.

$\mathrm{O}$ aumento significativo da $\mathrm{T}_{\max }$ na veia esplênica, observada nos pacientes operados, provavelmente resultou da redução da resistência à vazão e conseqüente maior facilidade de drenagem venosa. Esta situação torna possível que a AERD promova tanto a redução da congestão esplênica, quanto da pressão no interior das varizes esofágicas, reduzindo o perigo de sangramento nesta região.

\section{CONCLUSÃO}

Cotejando os resultados morfométricos e hemodinâmicos encontrados, observou-se que na EHE a AERD promove a redução do volume esplênico, sem alterar as condições hemodinâmicas arteriais do baço. Entretanto, os poucos estudos realizados a este respeito e a falta de sistematização na medida dos parâmetros, dificultam a real interpretação destes resultados, abrindo-se assim, perspectivas para novos estudos da fisiologia e fisiopatologia da circulação esplênica. 
Widman A, Oliveira IRS, Speranzini MB, Cerri GG, Saad WA, Gama-Rodrigues J. Alterações morfológicas e hemodinâmicas tardias no território esplênico de pacientes com esquistossomose mansônica hepatoesplênica pós-anastomose esplenorrenal distal. (Estudo com ultra-som Doppler)

Widman A, Oliveira IRS, Speranzini MB, Cerri GG, Saad WA, Gama-Rodrigues J. Late morphologic and hemodynamic changes in the splenic territory of patients with mansoni's hepatosplenic schistosomiasis after distal splenorenal shunt. (Ultrasonography-Doppler study). Arq Gastroenterol 2002;39(4):217-221.

ABSTRACT - Background - The distal splenorenal anastomosis (Warren's operation) has been indicated for the treatment of high digestive bleeding caused by esophagic varices because it would ideally reduce the venous pressure in the cardiotuberositary territory without changing the mesenteric-portal venous flow. However, the changes it produce in the splenic territory have not been fully understood. Aim - To appraise the late morphologic and hemodynamic changes in the splenic territory produced by the distal splenorenal anastomosis in patients with portal hypertension due to mansoni's hepatosplenic schistosomiasis complicated by esophagic bleeding. Method - Ultrasonography-Doppler study of the splenic region of 52 patients with portal hypertension due to mansoni's schistosomiasis and previous bleeding by esophagic varices. They were divided in two groups: 40 non operated upon and 12 with a distal splenorerenal anastomosis. The following parameters and indices were compared between the two groups: a) morphometric parameters (splenic artery and vein's diameter, splenic diameters (longitudinal, transversal and antero-posterior); b) velocimetric parameters of the splenic vessels (systolic peak velocity in the splenic artery, mean flow velocity in the splenic vein; c) biometric index of the spleen (longitudinal $x$ transversal); volumetric index of the spleen (longitudinal $x$ transversal $x$ anteroposterior x 0,523); hemodynamic indices of the splenic artery's impedance: pulsatility and resistivity. Results - The patients with distal splenorenal anastomosis showed: a) reduction in splenic indices: volumetric (non operated 903,83 $\pm 452,77 \mathrm{~cm}^{3} /$ distal splenorenal anastomosis $482,32 \pm 208,02 \mathrm{~cm}^{3}(46,64 \%)$ ) and biometric (non operated 138,14 $\pm 51,89 \mathrm{~cm}^{2} /$ distal splenorenal anastomosis $94,83 \pm 39,83 \mathrm{~cm}^{2}(33,35 \%)$ ); b) no change: splenic artery's diameter (non operated $0,57 \pm 0,16 \mathrm{~cm} /$ distal splenorenal anastomosis $0,57 \pm 0,23 \mathrm{~cm}$ ); velocity in the splenic artery non operated $107 \pm 42,02 \mathrm{~cm} / \mathrm{seg} /$ distal splenorenal anastomosis $89,81 \pm 41,20 \mathrm{~cm} / \mathrm{seg}$ ), resistivity (non operated $0,58 \pm 0,008 /$ distal splenorenal anastomosis $0,56 \pm 0,06$ ) and pulsatility (non operated $0,91 \pm 0,19 /$ distal splenorenal anastomosis $0,86 \pm 0,15$, splenic vein (non operated $1,10 \pm 0,30 \mathrm{~cm} /$ distal splenorenal anastomosis $1,19 \pm 0,29 \mathrm{~cm}$ ); c) increase: mean flow velocity in the splenic vein (non operated $20,54 \pm 8,45 \mathrm{~cm} / \mathrm{seg} /$ distal splenorenal anastomosis $27,83 \pm 9,29 \mathrm{~cm} / \mathrm{seg}$ ). Conclusions - The comparison of the ultrasonography Doppler results of the two groups of patient (non operated and distal splenorenal anastomosis) showed that in patients with distal splenorenal anastomosis there was a decrease of the volume of spleen; increase in the mean flow velocity in the splenic vein; no changes in the morphologic and hemodinamyc parameters of the splenic artery neither in its velocimetric indices.

HEADINGS - Spleen. Schistosomiasis mansoni. Hypertension portal. Anastomosis, surgical. Ultrasonography, Doppler.

\section{REFERÊNCIAS BIBLIOGRÁFICAS}

1. Abdel Wahab MF, el-Kady N, Arafa NM, Salama Z, Kotb AK, Nasef A, Abdou M, Hamam S, el-Din Hussein K. Distal splenorenal shunts (Warren's operation) Bmode and real time ultrasonographic assessment. Egypt J Bilharz 1979;6:21-4

2. Bessa SM, Helmy I, El-Sheikh O, Hamam SM, El-Kishen MA. The dista splenorenal shunt in patients with variceal bleeding due to schistosomal hepatic fibrosis. Surg Gynecol Obstet 1987;163:143-7.

3. Bogliolo L. Sobre o quadro anatômico do fígado na forma hepatoeplênica da esquistossomose mansônica. Hospital (Rio de janeiro) 1954;45(3):29-58

4. Bogliolo L. O peso do fígado e do baço na esquistossomose mansônica hepatoesplênica e na doença de Morgagni-Laennec. Rev Assoc Med Bras 1956;2:386-92.

5. Bolognesi M, Sacerdoti D, Merkel C, Gerunda G, Maffei-Faccioli A, Angeli P, Jemmolo RM, Bombonato G, Gatta A. Splenic Doppler impedance índices: influence of different portal hemodynamic conditions. Hepatology 1996;23:1035-40.

6. Breiman RS, Beck JW, Korobkin M, Glenny R, Akwari OE, Heaston DK, Moore AV, Ram PC. Volume determinations using computed tomography. AJR 1982;138:329-33.

7. DeOdorico I, Spaulding KA, Pretorius DH, Lev-Toaff AS, Bailey TB, Nelson TR. Normal splenic volumes estimated using three-dimensional ultrasonography. J Ultrasound Med 1999;18:231-6.

8. Ezzat FA, Abu-Elmagd KM, Aly IY, Aly MA, Fathy OM, El-Barbary MH, Bahgat OO, Salam AA, Kutner MH. Distal splenorenal shunt for management of variceal bleeding in patients with schistosomal hepatic fibrosis. Ann Surg 1986;204:566-73

9. Gilja OH, Thune N, Matre K, Hausken T, Ødegaard, Berstad A. In vitro evaluation of three-dimensional ultrasonography in volume estimation of abdominal organs. Ultrasound in Med Biol 1994;20:157-65.

10. Ishibashi H, Higuchi N, Shimamura R, Hirata Y, Kudo J, Niho Y. Sonographic assesment and grading of spleen size. J Clin Ultrasound 1991;19:21-5.

11. Kardel T, Holm HH, Rasmussen N, Mortesen T. Ultrasonic determination of liver and spleen volumes. Scand J Clin Lab Invest 1971;27:123-8.
12. Koga T, Morikawa Y. Ultrasonographic determination of the splenic size and its clinical usefulness in various liver diseases. Radiology 1975;115:157-61.

13. Lopes-Filho GJ, Haddad CM. Late clinical, biochemical, endoscopic and encephalographic evaluation of patients with schistosomal portal hypertension treated with distal splenorenal shunt. Int Surg 1998;83:42-7.

14. Magalhães AC. Evaluation of the permeability of distal splenorenal anastomosis by ultrasonography and angiography in hepatosplenic form of schistosomiasi mansoni. Rev Hosp Clin Fac Med Sao Paulo 1986;41:260-6.

15. Niederau C, Sonnenberg A, Müller JE, Erckenbrecht JF, Scolten T, Fritsch WP Sonographic measurements of the normal liver, spleen, pancreas and portal vein. Radiology 1983;149:537-40.

16. Nimeh W. New method for the determination of the size of the liver and spleen Am J Gastroenterol 1955;23:14-6.

17. Raia S, Silva AT, Lopes JD, Mies S, Oliveira e Silva A, Strauss E, Laudanna A Raia A, Silva LC. Selective portal decompression by distal splenorenal anastomosis for the treatment of esophageal bleeding varices in schistosomiasis mansoni. Rev Hosp Clin Fac Med Sao Paulo 1971;26:149-52.

18. Riccabona M, Nelson TR, Pretorius DH, Davidson TE. Distance and volume measurement using three-dimensional ultrasonography. J Ultrasound Med $1995 ; 14: 881-6$

19. Rosenbeg HK, Markowitz RI, Kolberg H, Park C, Hubbard A, Bellah RD. Normal splenic size in infants and children: sonographic measurements. AJR 1991;157:119-21

20. Saad WA, Yamin R, Velarde FUG, Chaib S, Parra OM, Bermann A, Vasconcelos E Descompressão seletiva do sistema portal no tratamento da hipertensão portal associada a varizes de esôfago sangrantes. Rev Assoc Med Bras 1977;23:165-8.

21. Warren WD, Zeppa R, Foman JJ. Selective transplenic decompression of gastroesophageal varices by distal spleno-renal shunt. Ann Surg 1967;166:437-55.

22. Zhang B, Lewis SM. Use of radionuclide scanning to estimate size of spleen in vivo. J Clin Pathol 1987:40:508-11. 